\title{
OPTIMAL BEAM STEERING ANGLES OF A SENSOR ARRAY FOR A MULTIPLE SOURCE SCENARIO
}

\author{
Sanghyouk Choi ${ }^{1}$, Joohwan Chun ${ }^{1}$, Inchan Paek ${ }^{2}$ and Jonghun Jang ${ }^{3}$ \\ ${ }^{1}$ Department of Electrical Engineering, KAIST, Daejeon, Korea \\ shchoilsclab.kaist.ac.kr \\ ${ }^{2}$ PGM Image Sensor Centre, Hanwha Thales, Kyunggi-do, Korea \\ ic.paek@hanwha.com \\ ${ }^{3}$ Agency for Defense Development, Daejeon, Korea
}

\begin{abstract}
We present the gradient and Hessian of the trace of the multivariate Cramér-Rao bound (CRB) formula for unknown impinging angles of plane waves with non-unitary beamspace measurements. . These gradient and Hessian can be used to find the optimal beamspace transformation matrix, i.e., the optimum beamsteering angles, using the Newton-Raphson iteration. These trace formulas are particularly useful to deal with the multiple source senario. We also show the mean squred error (MSE) performance gain of the optimally steered beamspace measurements compared with the usuall DFT steered measurements, when the angle of arrivals (AOAs) are estimated with stochastic maximum likelihood (SMLE) algorithm.
\end{abstract}

\section{KEYWORDS}

Cramér-Rao bound, beamspace transformation, correlated noise, DFT beams, subarray formation

\section{INTRODUCTION}

The angle of arrival (AOA) estimation problem arises a wide variety of applications dealing with electromagnetic or sound waves. Therefore, AOA estimation has been one of the most active research topics for the past several decades, and various algorithms such as the interferometry, monopulse, MUSIC, ESPRIT and maximum likelihood estimation (MLE) have been devised. A related problem of theoretical importance is to get the Cramér-Rao bound (CRB), i.e, a lower bound of the mean squared error (MSE) for the AOA estimation, which also has been studied by many researchers. However, what has been missing in this direction of research is the investigation of the effect of controllable parameters on the CRB. In this paper, we derive the optimal beam directions that minimizes the CRB for the AOA estimation problem in the presence of multiple impinging plane waves. We also show that the resulting optimal directions give a lower MSE through Monte-Carlo simulation.

The angle of arrival (AOA) estimation may be carried out either in the sensor element space or in the beam space. Although accurate angle estimation requires an array with a large number of

Jan Zizka et al. (Eds) : CCSIT, SIPP, AISC, CMCA, SEAS, CSITEC, DaKM, PDCTA, NeCoM - 2016

pp. 141-150, 2016. C CS \& IT-CSCP 2016

DOI : $10.5121 /$ csit.2016.60113 
sensors in general, direct utilization of all sensors is impractical. The reason is that manipulation of full multi-channel digital data incurs high computational burden, let alone the high hardware cost for down-conversion and digitization of every sensor measurement signal. To alleviate the difficulty, a dimension reduction matrix $B \in \mathrm{X}^{M \times K}, K<M$, may be used to transform an element space (ES) measurement vector $z \in \mathrm{X}^{M \times 1}$ to a beamspace (BS) measurement vector $B^{H} z \in \mathrm{X}^{K \times 1}$, where superscript $H$ denotes the conjugate transpose. The BS transformation matrix, $B$ may be designed under different criteria, for example, to cover a given spatial sector [5], to maximize the signal to noise ratio (SNR) in the sector [11], to minimize the interfering power [10], to minimize the Cramér-Rao bound (CRB) [1], or simply to ease the implementation by employing the discrete Fourier transformation (DFT) [13], [2], [12]. Apart from the DFTbased transformation, the above-mentioned $B$ will have arbitrary complex-numbered elements, and therefore, they will incur higher hardware cost than the case with unit-modulus complex numbers, if $B$ is to be implemented with analog parts.

If we design $B$ with unit-modulus elements, it admits a simpler implementation using phaseshifters only. In other words, we wish to steer the beams tactfully, in a different manner from the usual orthogonal DFT beams, so that the CRB for the angle estimation is the minimum. A block diagram of the proposed transformation is shown in Fig 1.

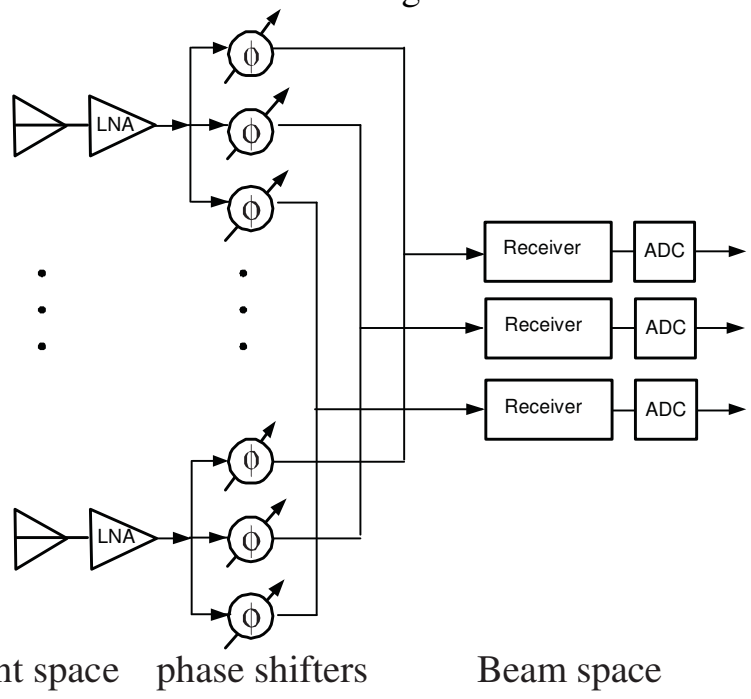

Figure1: Beamspace transformation with sphase-shifters only $(\mathrm{K}=3)$

Now the CRB formula in [9] cannot be used to find the optimum steering angles because $B$ is not unitary and therefore the measurement noise is spatially correlated. Furthermore, the prewhitening technique $[4,6,7]$ cannot be used because the pre-whitening matrix will not be an explicit parametric function of the steering angles, and therefore does not admit explict differentiations.

\section{REVIEW OF THE CRB FOR BEAM SPACE MEASUREMENT}

Consider the measurement vector $z \in \mathrm{X}^{M \times 1}$ of an array with $M$ sensors, 


$$
z=A s+n, \quad A=\left[a\left(\theta_{1}\right), \Lambda, a\left(\theta_{N}\right)\right], \quad s=\left[s_{1}, \Lambda, s_{N}\right]^{T}
$$

where the $n$th column $a\left(\theta_{n}\right)$ of $A \in \mathrm{X}^{M \times N}$ is the array response vector for the $n$th plane-wave signal $s_{n}$ impinging at the angle $\theta_{n}$, superscript $T$ denotes the transpose, and $n: C N\left(0, \sigma_{n}^{2} I\right)$ denotes the spatially and temporally uncorrelated measurement noise vector. Then, the stochastic $\mathrm{CRB}$ of $\boldsymbol{\theta}=\left[\theta_{1}, \Lambda, \theta_{N}\right]^{T}$ is given by [9]

$$
C R B(\boldsymbol{\theta})=\frac{\sigma_{n}^{2}}{2 L}\left\{\operatorname{Re}\left[\left(D^{H} \Pi_{A}^{\perp} D\right) \varepsilon\left(P A^{H} R^{-1} A P\right)^{T}\right]\right\}^{-1}
$$

where $R=E z z^{H}=A P A^{H}+\sigma_{n}^{2} I, \quad P=E s s^{H}, \quad \Pi_{A}^{\perp}=I-A\left(A^{H} A\right)^{-1} A^{H}, \quad D=\left[d_{1}, \Lambda, d_{n}\right]$, $d_{n}=\frac{d a\left(\theta_{n}\right)}{d \theta_{n}}, L$ is the number of snapshots, $\operatorname{Re}(\cdot)$ is the real-part operator, and $\varepsilon$ denotes the Hadamard-Schur product.

Let $B=\left[a\left(\theta_{b 1}\right), \Lambda, a\left(\theta_{b K}\right)\right] \in \mathrm{X}^{M \times K}$ denote a BS transformation matrix, towards a steering angle $\boldsymbol{\theta}_{b}=\left[\theta_{b 1}, \Lambda, \theta_{b K}\right]^{T}$. Then the resulting dimension reduced measurement will be

$$
z_{B}=B^{H} A s+B^{H} n
$$

In general, the noise vector $B^{H} n$ is correlated, which may be pre-whitened as $z_{B}=U B^{H} A s+U B^{H} n$, where $U$ is a Hermitian square root matrix of $\left(B^{H} B\right)^{-1}$. Now the stochastic BS CRB can be expressed as

$$
C R B_{B}\left(\boldsymbol{\theta}, \boldsymbol{\theta}_{b}\right)=\frac{\sigma_{n}^{2}}{2 L}\left\{\operatorname{Re}\left(F \varepsilon G^{T}\right)\right\}^{-1},
$$

where $F=D^{H} B U^{H} \Pi_{U B^{H}}^{\perp} U B^{H} D, G=P A^{H} B U^{H} R^{-1} U B^{H} A P$ and $R=U B^{H} A P A^{H} B U^{H}+\sigma_{n}^{2} I$. However, $U$ is a function of $\theta_{b k}$ whose explicit form is not available, and therefore, we cannot minimize (4) with respect to $\theta_{b k}$. The following lemma [12] gives a direct CRB formula for (3), not resorting to pre-whitening.

Lemma 1 For the BS measurement model in (3), the CRB for $\boldsymbol{\theta}$ is given as

$$
C R B_{B}\left(\boldsymbol{\theta}, \boldsymbol{\theta}_{b}\right)=\frac{1}{2 L}\left\{\operatorname{Re}\left(F \varepsilon G^{T}\right)\right\}^{-1}
$$

where $F=D^{H} \Pi_{\Pi_{B} A}^{\perp} B R^{-1} B^{H} D, G=\left(P A^{H} B R^{-1} B^{H} A P\right)^{T}$ and $R=B^{H} A P A^{H} B+\sigma_{n}^{2} B^{H} B$. 


\section{GRADIENT AND HESSIAN OF THE TRACE OF THE CRB}

Assuming that multiple sources impinge on an array of sensors at angles, $\boldsymbol{\theta}=\left[\theta_{1}, \Lambda, \theta_{N_{s}}\right]^{T}$, we are interested in finding the optimal steering angles $\boldsymbol{\theta}_{b}$ for the BS transformation matrix $B=\left[a\left(\theta_{b 1}\right), \Lambda, a\left(\theta_{b k}\right)\right]$ such that

$$
\hat{\boldsymbol{\theta}}_{b}=\underset{\theta_{b}}{\operatorname{argmin}} \operatorname{tr}\left[C R B\left(\boldsymbol{\theta}, \boldsymbol{\theta}_{b}\right)\right] .
$$

Since $\operatorname{tr}\left[C \operatorname{RB}\left(\boldsymbol{\theta}, \boldsymbol{\theta}_{b}\right)\right]$ is a nonlinear function of $\boldsymbol{\theta}_{b}$, the minimization needs to be carried out numerically.

We shall use the Newton-Raphson iteration which needs the gradient $\operatorname{Vtr}\left[\operatorname{CRB}\left(\boldsymbol{\theta}, \boldsymbol{\theta}_{b}\right)\right]$ and Hessian $H\left(\operatorname{tr}\left[C R B\left(\boldsymbol{\theta}, \boldsymbol{\theta}_{b}\right)\right]\right)$ of $\operatorname{tr}\left[\mathbf{C R B}\left(\boldsymbol{\theta}, \boldsymbol{\theta}_{b}\right)\right]$.

They are provided in the following lemmas.

Lemma 2 The $i$ th element of the gradient vector, $\nabla \operatorname{tr}\left[C R B\left(\boldsymbol{\theta}, \boldsymbol{\theta}_{b}\right)\right]$ is given by

$$
\frac{\partial \operatorname{tr}\left[C R B\left(\boldsymbol{\theta}, \boldsymbol{\theta}_{b}\right)\right]}{\partial \theta_{b i}}=-\frac{1}{2 N} \operatorname{tr}\left[\operatorname{Re}(F \varepsilon G)^{-1} \cdot \operatorname{Re}\left(U_{i} \varepsilon G+F \varepsilon V_{i}\right) \cdot \operatorname{Re}(F \varepsilon G)^{-1}\right]
$$

where

$$
\begin{aligned}
F & =\left(D^{H} \Pi_{\Pi_{B} A}^{\perp} B R^{-1} B^{H} D\right) \\
G & =\left(P A^{H} B R^{-1} B^{H} A P\right)^{T} \\
U_{i} & =\frac{\partial F}{\partial \theta_{b i}}=\left(D^{H} H_{i} B R^{-1} B^{H} D\right)+\left(D^{H} \Pi_{\Pi_{B} A}^{\perp} C_{i} R^{-1} B^{H} D\right) \\
& +\left(D^{H} \Pi_{\Pi_{B} A}^{\perp} B R^{-1} C_{i}^{H} D\right)+\left(D^{H} \Pi_{\Pi_{B} A}^{\perp} B L_{i} B^{H} D\right) \\
V_{i} & =\frac{\partial G}{\partial \theta_{b i}}=\left(P A^{H} C_{i} R^{-1} B^{H} A P\right)^{T}+\left(P A^{H} B R^{-1} C_{i}^{H} A P\right)^{T}+\left(P A^{H} B L_{i} B^{H} A P\right)^{T} \\
C_{i} & =\frac{\partial B}{\partial \theta_{i}} \\
L_{i} & =\frac{\partial R^{-1}}{\partial \theta_{b i}}=-R^{-1} G_{i} R^{-1} \\
H_{i} & =\frac{\partial \Pi_{\Pi_{B} A}^{\perp}}{\partial \theta_{b i}}=\frac{\partial}{\partial \theta_{b i}}\left(I-\Pi_{B} A\left(A^{H} \Pi_{B} A\right)^{-1} A^{H} \Pi_{B}\right) \\
& =-\frac{\partial}{\partial \theta_{b i}} \Pi_{B} A\left(A^{H} \Pi_{B} A\right)^{-1} A^{H} \Pi_{B}
\end{aligned}
$$




$$
\begin{aligned}
& =-O_{i} A\left(A^{H} \Pi_{B} A\right)^{-1} A^{H} \Pi_{B}-\Pi_{B} A\left(A^{H} \Pi_{B} A\right)^{-1} A^{H} O_{i}-\Pi_{B} A P_{i} A^{H} \Pi_{B} \\
P_{i} & =\frac{\partial}{\partial \theta_{b i}}\left(A^{H} \Pi_{B} A\right)^{-1}=-\left(A^{H} \Pi_{B} A\right)^{-1}\left(A^{H} O_{i} A\right)\left(A^{H} \Pi_{B} A\right)^{-1} \\
O_{i} & =\frac{\partial}{\partial \theta_{b i}} \Pi_{B}=C_{i}\left(B^{H} B\right)^{-1} B^{H}+B Q_{i} B^{H}+B\left(B^{H} B\right)^{-1} C_{i}^{H} \\
Q_{i} & =\frac{\partial}{\partial \theta_{b i}}\left(B^{H} B\right)^{-1}=-\left(B^{H} B\right)^{-1}\left(C_{i}^{H} B+B^{H} C_{i}\right)\left(B^{H} B\right)^{-1} \\
G_{i} & =\frac{\partial R}{\partial \theta_{b i}}=C_{i}^{H} A P A^{H} B+B^{H} A P A^{H} C_{i}+\sigma_{n}^{2} C_{i}^{H} B+B^{H} C_{i}
\end{aligned}
$$

Proof. The proof of this lemma is omitted because it is tedious but straightforward, utilizing matrix differentiation rules.

Lemma 3 The $(i, j)$ th element of the Hessian matrix $H\left(\operatorname{tr}\left[C R B\left(\boldsymbol{\theta}, \boldsymbol{\theta}_{b}\right)\right]\right)$ is given by

$$
\begin{aligned}
& \frac{\partial \operatorname{tr}\left[C R B\left(\theta, \theta_{b}\right)\right]}{\partial \theta_{b i} \partial \theta_{b j}}=-\frac{1}{2 N} \operatorname{tr}\left[\operatorname{Re}\left(\frac{\partial Q}{\partial \theta_{b j}}\right)^{-1} \cdot \operatorname{Re}\left(\frac{\partial Q}{\partial \theta_{b i}}\right) \cdot \operatorname{Re}(Q)^{-1}\right. \\
& \left.\quad+\operatorname{Re}(Q)^{-1} \cdot \operatorname{Re}\left(\frac{\partial Q}{\partial \theta_{b i} \partial \theta_{b j}}\right) \cdot \operatorname{Re}(Q)^{-1}+\operatorname{Re}(Q)^{-1} \cdot \operatorname{Re}\left(\frac{\partial Q}{\partial \theta_{b i}}\right) \cdot \operatorname{Re}\left(\frac{\partial Q}{\partial \theta_{b j}}\right)^{-1}\right]
\end{aligned}
$$

where $Q=F \varepsilon G$ and

$$
\begin{aligned}
& \operatorname{Re}\left(\frac{\partial Q}{\partial \theta_{b j}}\right)^{-1}=-\operatorname{Re}(Q)^{-1} \cdot \operatorname{Re}\left(\frac{\partial Q}{\partial \theta_{b j}}\right) \cdot \operatorname{R} e(Q)^{-1} \\
& \operatorname{Re}\left(\frac{\partial Q}{\partial \theta_{b i}}\right)=\operatorname{Re}\left(U_{i} \varepsilon G+F \varepsilon V_{i}\right) \\
& \operatorname{Re}\left(\frac{\partial Q}{\partial \theta_{b i} \partial \theta_{b j}}\right)=\operatorname{Re}\left(U_{i, j} \varepsilon G+U_{i} \varepsilon V_{j}+U_{j} \varepsilon V_{i}+F \varepsilon V_{i, j}\right)
\end{aligned}
$$

and

$$
\begin{aligned}
& U_{i, j}=\frac{\partial U_{i}}{\partial \theta_{b j}}=\left(D^{H} H_{i, j} B R^{-1} B^{H} D\right)+\left(D^{H} H_{i} C_{j} R^{-1} B^{H} D\right) \\
& +\left(D^{H} H_{i} B L_{j} B^{H} D\right)+\left(D^{H} H_{i} B R^{-1} C_{j}{ }^{H} D\right) \\
& +\left(D^{H} H_{j} C_{i} R^{-1} B^{H} D\right)+\left(D^{H} \Pi_{\Pi_{B} A}^{\perp} C_{i, j} R^{-1} B^{H} D\right) \\
& +\left(D^{H} \Pi_{\Pi_{B} A}^{\perp} C_{i} L_{j} B^{H} D\right)+\left(D^{H} \Pi_{\Pi_{B}{ }_{A}}^{\perp} C_{i} R^{-1} C_{j}{ }^{H} D\right)
\end{aligned}
$$




$$
\begin{aligned}
& +\left(D^{H} H_{j} B R^{-1} C_{i}^{H} D\right)+\left(D^{H} \Pi_{\Pi_{B}{ }_{A}}^{\perp} C_{j} R^{-1} C_{i}^{H} D\right) \\
& +\left(D^{H} \Pi_{\Pi_{B}{ }_{A}}^{\perp} B L_{j} C_{i}{ }^{H} D\right)+\left(D^{H} \Pi_{\Pi_{B}{ }^{A}}^{\perp} B R^{-1} C_{i, j}{ }^{H} D\right) \\
& +\left(D^{H} H_{j} B L_{i} B^{H} D\right)+\left(D^{H} \Pi_{\Pi_{B} A}^{\perp} C_{j} L_{i} B^{H} D\right) \\
& +\left(D^{H} \Pi_{\Pi_{B}{ }_{A}}^{\perp} B L_{i, j} B^{H} D\right)+\left(D^{H} \Pi_{\Pi_{B}{ }^{A}}^{\perp} B L_{i} C_{j}{ }^{H} D\right) \\
& V_{i, j}=\frac{\partial V_{i}}{\partial \theta_{b j}}=\left(P A^{H} C_{i, j} R^{-1} B^{H} A P\right)^{T}+\left(P A^{H} C_{i} L_{j} B^{H} A P\right)^{T} \\
& +\left(P A^{H} C_{i} R^{-1} C_{j}{ }^{H} A P\right)^{T}+\left(P A^{H} C_{j} L_{i} B^{H} A P\right)^{T} \\
& +\left(P A^{H} B L_{i, j} B^{H} A P\right)^{T}+\left(P A^{H} B L_{i} C_{j}{ }^{H} A P\right)^{T} \\
& +\left(P A^{H} C_{j} R^{-1} C_{i}^{H} A P\right)^{T}+\left(P A^{H} B L_{j} C_{i}^{H} A P\right)^{T} \\
& +\left(P A^{H} B R^{-1} C_{i, j}{ }^{H} A P\right)^{T} \\
& C_{i, j}=\frac{\partial B}{\partial \theta_{b i} \partial \theta_{b j}}=0 \\
& H_{i, j}=\frac{\partial}{\partial \theta_{b i} \partial \theta_{b j}} \Pi_{\Pi_{B} A}^{\perp}=-O_{i, j} A\left(A^{H} \Pi_{B} A\right)^{-1} A^{H} \Pi_{B}-O_{i} A P_{j} A^{H} \Pi_{B} \\
& -O_{i} A\left(A^{H} \Pi_{B} A\right)^{-1} A^{H} O_{j}-O_{j} A\left(A^{H} \Pi_{B} A\right)^{-1} A^{H} O_{i} \\
& -\Pi_{B} A P_{j} A^{H} O_{i}-\Pi_{B} A\left(A^{H} \Pi_{B} A\right)^{-1} A^{H} O_{i, j} \\
& -O_{j} A P_{i} A^{H} \Pi_{B}-\Pi_{B} A P_{i, j} A^{H} \Pi_{B}-\Pi_{B} A P_{i} A^{H} O_{j} \\
& P_{i, j}=\frac{\partial}{\partial \theta_{b j}} P_{i}=-P_{j}\left(A^{H} O_{i} A\right)\left(A^{H} \Pi_{B} A\right)^{-1} \\
& -\left(A^{H} \Pi_{B} A\right)^{-1}\left(A^{H} O_{i, j} A\right)\left(A^{H} \Pi_{B} A\right)^{-1}-\left(A^{H} \Pi_{B} A\right)^{-1}\left(A^{H} O_{i} A\right) P_{j} \\
& O_{i, j}=\frac{\partial}{\partial \theta_{b j}} O_{i}=C_{i, j}\left(B^{H} B\right)^{-1} B^{H}+C_{i} Q_{j} B^{H}+C_{i}\left(B^{H} B\right)^{-1} C_{j}{ }^{H}+C_{j} Q_{i} B^{H} \\
& +B Q_{i, j} B^{H}+B Q_{i} C_{j}{ }^{H}+C_{j}\left(B^{H} B\right)^{-1} C_{i}{ }^{H}+B Q_{j} C_{i}{ }^{H}+B\left(B^{H} B\right)^{-1} C_{i, j}{ }^{H} \\
& Q_{i, j}=\frac{\partial}{\partial \theta_{b j}} Q_{i}=-Q_{j}\left(C_{i}^{H} B+B^{H} C_{i}\right)\left(B^{H} B\right)^{-1}-\left(B^{H} B\right)^{-1}\left(C_{i}^{H} B+B^{H} C_{i}\right) Q_{j} \\
& -\left(B^{H} B\right)^{-1}\left(C_{i}^{H} C_{j}+C_{j}{ }^{H} C_{i}\right)\left(B^{H} B\right)^{-1} \\
& L_{i, j}=\frac{\partial R^{-1}}{\partial \theta_{b i} \partial \theta_{b j}}=-\frac{\partial R^{-1}}{\partial \theta_{b j}} G_{i} R^{-1}-R^{-1} \frac{\partial G_{i}}{\partial \theta_{b j}} R^{-1}-R^{-1} G_{i} \frac{\partial R^{-1}}{\partial \theta_{b j}} \\
& =-L_{j} G_{i} R^{-1}-R^{-1} G_{i, j} R^{-1}-R^{-1} G_{i} L_{j}
\end{aligned}
$$




$$
\begin{aligned}
G_{i, j} & =\frac{\partial G_{i}}{\partial \theta_{j}}=C_{i, j}{ }^{H} A P A^{H} B+C_{i}{ }^{H} A P A^{H} C_{j}+C_{j}{ }^{H} A P A^{H} C_{i}+B^{H} A P A^{H} C_{i, j} \\
& +\sigma_{n}^{2} C_{i, j}{ }^{H} B+\sigma_{n}^{2} C_{i}{ }^{H} C_{j}+\sigma_{n}^{2} C_{j}{ }^{H} C_{i}+\sigma_{n}^{2} B^{H} C_{i, j}
\end{aligned}
$$

Proof. The proof of this lemma is omitted because it is tedious but straightforward, utilizing matrix differentiation rules.

The gradient in (7) and Hessian in (9) contain the unknown parameter $\theta$, which we are ultimately interested to find, and therefore, cannot be directly used for Newton-Raphson iteration for finding $\boldsymbol{\theta}_{b}$. In reality, however, information on the interval $\Theta_{s}$, where $\theta$ must be in, is usually available to us, for example from a surveillance radar, and therefore, it is appropriate to use the averaged CRB

$$
\overline{C R B}\left(\Theta_{s}, \boldsymbol{\theta}_{b}\right)=\int_{\Theta_{s}} C R B\left(\theta, \boldsymbol{\theta}_{b}\right) d \theta \approx \sum_{\theta_{i} \in \Theta_{s}} C R B\left(\theta_{i}, \boldsymbol{\theta}_{b}\right) \cdot \Delta \theta .
$$

This, in turns, gives the following averaged gradient and Hessian, after interchanging the integration and differentiation:

$$
\begin{aligned}
& \nabla\left[\operatorname{tr} \overline{C R B}\left(\Theta_{s}, \boldsymbol{\theta}_{b}\right)\right] \approx \sum_{\theta_{i} \in \Theta_{s}} \nabla\left[\operatorname{trCRB}\left(\theta_{i}, \boldsymbol{\theta}_{b}\right)\right] \cdot \Delta \theta, \\
& H\left[\operatorname{tr}\left(\overline{C R B}\left(\Theta_{s}, \boldsymbol{\theta}_{b}\right)\right)\right] \approx \sum_{\theta_{i} \in \Theta_{s}} H\left[\operatorname{tr}\left(C R B\left(\theta_{i}, \boldsymbol{\theta}_{b}\right)\right)\right] \cdot \Delta \theta .
\end{aligned}
$$

Therefore, the optimal $\hat{\boldsymbol{\theta}}_{b}$ is found by

$$
\hat{\boldsymbol{\theta}}_{i+1}=\hat{\boldsymbol{\theta}}_{i}-\mu\left[H\left\{\operatorname{tr}\left(\overline{C R B}\left(\Theta_{s}, \hat{\boldsymbol{\theta}}_{i}\right)\right)\right\}\right]^{-1} \nabla\left[\operatorname{tr} \overline{C R B}\left(\Theta_{s}, \hat{\boldsymbol{\theta}}_{i}\right)\right]
$$

where $\hat{\boldsymbol{\theta}}_{i}=\left[\hat{\theta}_{b 1}, \Lambda, \hat{\theta}_{b k}\right]_{i}^{T}$

\section{Simulation RESUltS}

For a low-angle ship-borne radar, the target reflection usually returns back to the radar via two pathes - the direct path and the sea-surface reflection path [13, 14]. Although the AOA of the direct path is well-defined (for a continuous tracking radar), the AOA of the reflection path cannot be accurately predictable due to the diffuse refelction from rough sea surface. We shall consider a tracking radar with three beams, and obtain the optimal beam steering angles for this multipath senario.

First, we compare the CRBs of the proposed BS processing, with the DFT-based BS processing and the ES processing. Fig. 2 is the case when $M=16, K=3, L=6$ and $S N R=10 d B$. It is assumed that $\theta_{1}=1^{\circ}$ and $\theta_{2} \in \Theta_{s}$ where $\Theta_{s}=\left[-1.3^{\circ},-1.1^{\circ}\right]$. In this case, the computed optimal steering angles are $\theta_{b 1}=2.7266^{\circ}, \theta_{b 2}=-0.6966^{\circ}$ and $\theta_{b 3}=-2.3956^{\circ}$, while the DFT-based steering angles are $\theta_{b 1}=-7.1808^{\circ}, \theta_{b 2}=0^{\circ}$ and $\theta_{b 3}=7.1808^{\circ}$. The CRB of the proposed BS 
processing is not only lower than that of the DFT-based BS processing, but also close to that of the ES processing throughout the interval $\Theta_{s}$.

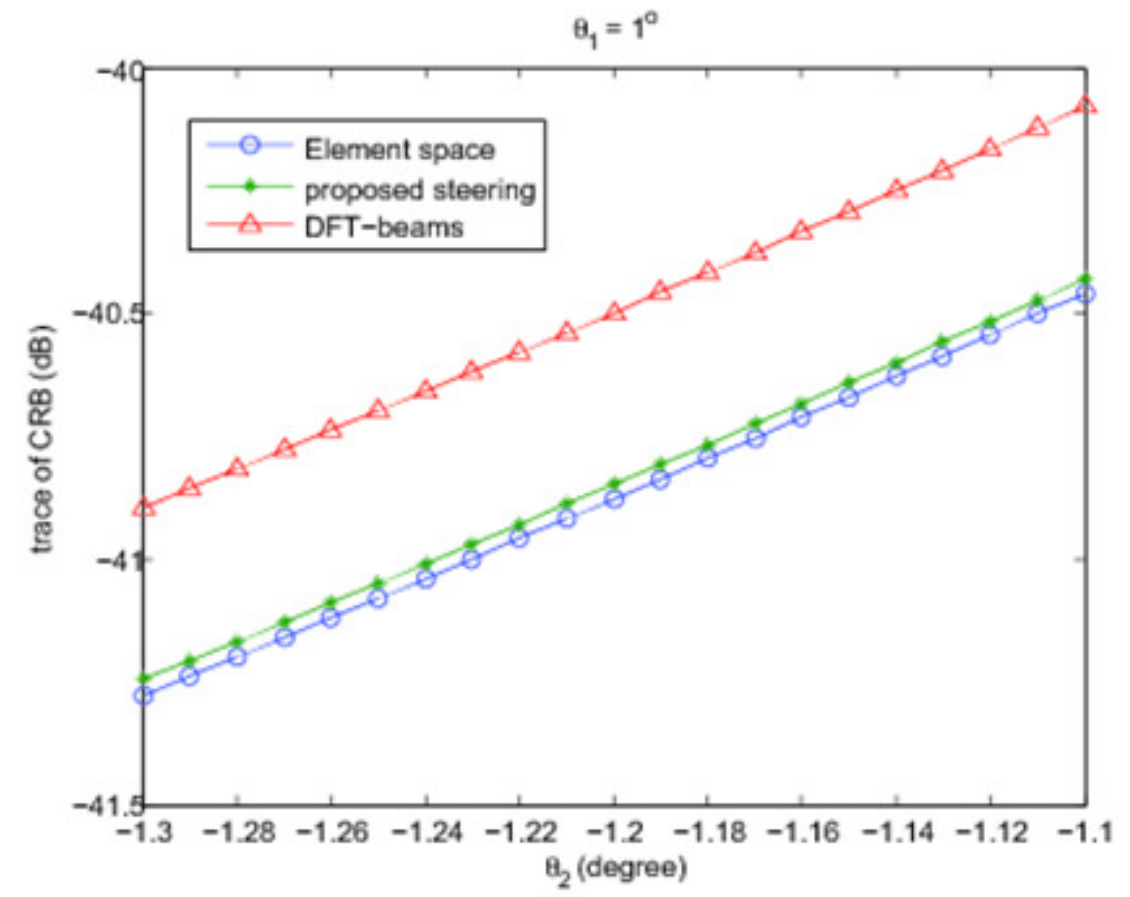

Figure 2: Comparison of the CRB with the proposed steering to that with the DFT-beam

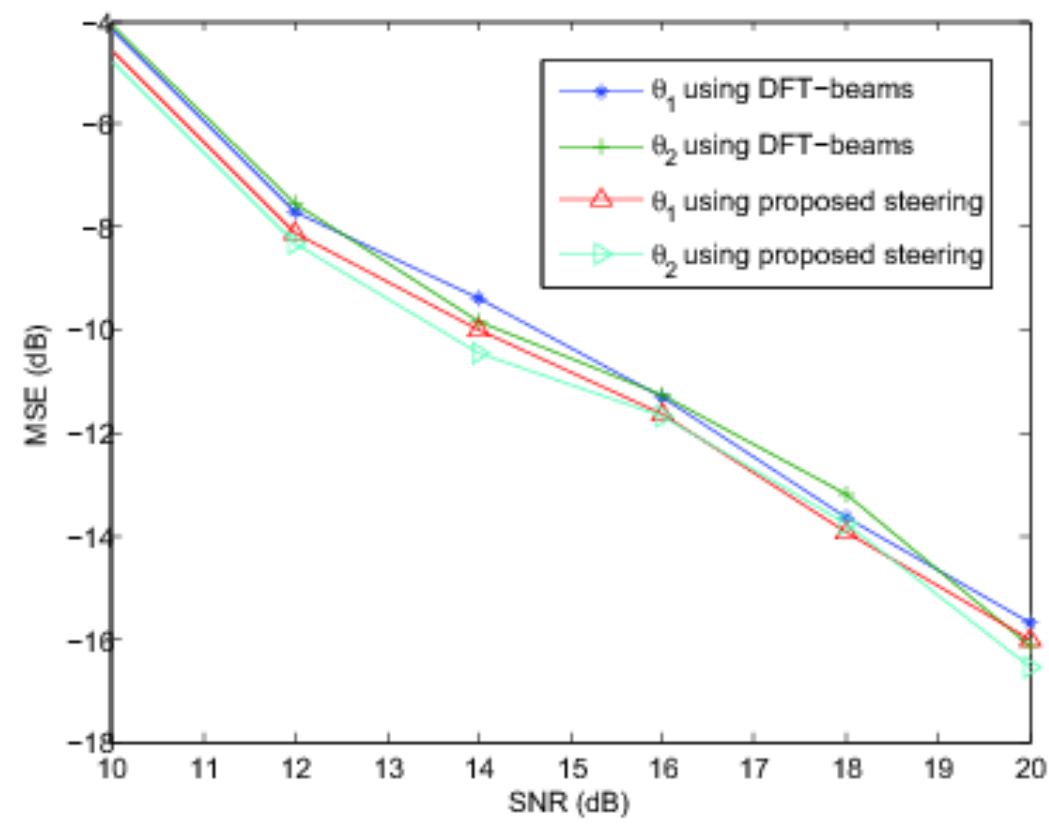

Figure 3: Comparison of the MSE with the proposed steering to that with the DFT beam

Second, we use the stochastic maximum likelihood (SMLE) method [8] 


$$
\begin{aligned}
& \theta^{i+1}=\theta^{i}-\mu_{i} H^{-1}\left(\theta^{i}\right) V^{\prime}\left(\theta^{i}\right) \\
& \left.V^{\prime}\left(\theta^{i}\right)=2 \operatorname{Re}\left(\operatorname{Diag} \mid g\left(\theta^{i}\right)^{H} \hat{R} P_{a_{s}}^{\perp} d\left(\theta^{i}\right)\right]\right) \\
& H\left(\theta^{i}\right)=2 \hat{\sigma}^{2} \operatorname{Re}\left\{d\left(\theta^{i}\right)^{H} P_{a_{s}}^{\perp} d\left(\theta^{i}\right) \cdot g^{H}\left(\theta^{i}\right) \hat{R} g\left(\theta^{i}\right)\right\} \\
& g\left(\theta^{i}\right)=a_{s}\left[\left(a_{s}^{H} \hat{R} a_{s}\right)^{-1}-\hat{\sigma}^{-2}\left(\theta^{i}\right)\left(a_{s}^{H} a_{s}\right)^{-1}\right] \\
& \hat{\sigma}^{2}\left(\theta^{i}\right)=\frac{1}{M-N} \operatorname{Tr}\left\{P_{a_{s}}^{\perp} \hat{R}\right\} .
\end{aligned}
$$

Here $a_{s}=U B^{H} a\left(\theta^{i}\right)$ is evaluated for two different $B \mathrm{~s}$; the proposed one and the DFT-based one. Mean squared errors (MSEs) of the two estimates for 100 trials are compared in Fig. 3.

\section{CONCLUSIONS}

We have used the non-unitary beamspace CRB formula [3] to derive the gradient and Hessian of the trace of the CRB. Then applying these gradient and Hessian to a three-beam array antenna, we have found a set of three optimum beamsteering angles, for the case of two impinging plane waves, where one AOA can be rather accurately predictable, while the other has a large uncertainty. The MSE with the SMLE AOA estimation with the resulting optimum three-beam array is shown to be lower than the MSE with orthogonal DFT-beam AOA estimation.

\section{ACKNOWLEDGEMENTS}

This work was supported in part by Hanwha Thales in 2014-2015.

\section{REFERENCES}

[1] Sören Anderson. On optimal dimension reduction for sensor array signal processing. Signal Processing, 30(2):245-256, 1993.

[2] Russell D Brown, Richard A Schneible, Michael C Wicks, Hong Wang, and Yuhong Zhang. Stap for clutter suppression with sum and difference beams. IEEE Trans. Aerosp. Electron. Syst., 36(2):634646, 2000.

[3] Sanghyuck Choi, Joohwan Chun, Inchan Paek, and Jonghun Jang. A stochastic crb for non-unitary beam-space transformations and its application to optimal steering angle design. IEEE Signal Process. Lett., 22(11):2014-2018, 2015.

[4] Jonny Eriksson and Mats Viberg. Adaptive data reduction for signals observed in spatially colored noise. Signal processing, 80(9):1823-1831, 2000.

[5] Philippe Forster and Georges Vezzosi. Application of spheroidal sequences to array processing. In Acoustics, Speech and Signal Processing, IEEE International Conference on ICASSP'87., volume 12, pages 2268-2271. IEEE, 1987.

[6] Aboulnasr Hassanien, Sherif Abd Elkader, Alex B Gershman, and Kon Max Wong. Convex optimization based beam-space preprocessing with improved robustness against out-of-sector sources. IEEE Trans. Signal Process.,, 54(5):1587-1595, 2006. 
[7] Minghui $\mathrm{Li}$ and Yilong Lu. Dimension reduction for array processing with robust interference cancellation. IEEE Trans. Aerosp. Electron. Syst., 42(1):103-112, 2006.

[8] Björn Ottersten, Mats Viberg, Petre Stoica, and Arye Nehorai. Exact and large sample maximum likelihood techniques for parameter estimation and detection in array processing. Springer, 1993.

[9] Petre Stoica, Erik G Larsson, and Alex B Gershman. The stochastic CRB for array processing: A textbook derivation. IEEE Signal Process. Lett., 8(5):148-150, 2001.

[10] Barry Van Veen and Richard A Roberts. Partially adaptive beamformer design via output power minimization. IEEE Trans. Acoust., Speech, Signal Process., 35(11):1524-1532, 1987.

[11] Barry Van Veen and Bruce Williams. Structured covariance matrices and dimensionality reduction in array processing. In Spectrum Estimation and Modeling, 1988., Fourth Annual ASSP Workshop on, pages 168-171. IEEE, 1988.

[12] Eunjung Yang, Ravi Adve, and Joohwan Chun. Hybrid direct data domain sigma-delta space-time adaptive processing algorithm in non-homogeneous clutter. IET Radar, Sonar and Navigation, 4(4):611-625, 2010.

[13] Michael D Zoltowski and Ta-Sung Lee. Maximum likelihood based sensor array signal processing in the beamspace domain for low angle radar tracking. IEEE Trans. Signal Process., 39(3):656-671, 1991.

[14] Dongmin Park, Eunjung Yang, Soyeon Ahn, Joohwan Chun, Adaptive beamforming for low-angle target tracking under multipath interference, IEEE Trans on Aerosp. Electron Syst. 50(4), pp. 25642577, 2014. 